\title{
An essential role of astrocytic mGluR5 in the somatosensory cortex in regulation of synaptogenesis and neuropathic pain
}

\author{
Yosuke Danjo ${ }^{1}$, Yukiho Hirayama ${ }^{1}$, Eiji Shigetomi ${ }^{1}$, Keisuke Shibata ${ }^{1}$, Kenta Takanashi ${ }^{1}$, \\ Youichi Shinozaki ${ }^{1}$, Sung Kwang Kim², Junichi Nabekura ${ }^{2}$, Schuichi Koizumi ${ }^{1}$ \\ ${ }^{I}$ Neuropharmacology, Interdisciplinary Graduate School of Medicine, University of Yamanashi, Japan, ${ }^{2}$ Division of \\ Homeostatic Development, National Institute for Physiological Sciences, Japan
}

Background: Astrocytes control neuronal activity and connectivity, and serve as the synaptogenesis inducer. Metabotropic glutamate receptor 5 (mGluR5) is a key molecule which detects neurotransmitter, glutamate, and generates astrocytic $\mathrm{Ca} 2$ + signaling. mGluR5 expression is limited in immature astrocytes, is absent in mature. Interestingly, astrocytic mGluR5 re-appears in neuronal disorders, such as stroke, Alzheimer's disease, epilepsy and neuropathic pain. We recently found that neuropathic pain, a chronic pain arising from peripheral nerve injury and often showing mechanical allodynia, was induced by activated astrocytes in the primary somatosensory cortex (S1). Astrocytes became synaptogenic phenotype in response to peripheral nerve injury, and rewired S1 cortical networks, thereby leading to mechanical allodynia. Such synaptogenic astrocytes express mGluR5, but its causative roles in synaptogenesis remain unknown. Here, we show that re-appearance of mGluR5 in S1 astrocytes is required for synaptogenesis and the pathogenesis of mechanical allodynia.

Methods: Mechanical allodynia induced by partial sciatic nerve ligation (PSNL), was assessed by von Frey test. Protein expression was investigated by immunohistochemistry and westernblotting. Astrocytic $\mathrm{Ca} 2+$ excitability was imaged by a 2-photon microscopy. To investigate the role of astrocytic mGluR5, we made astrocyte-specific mGluR5-deficient mice (astro-mGluR5-KO) by crossing GLAST-CreERT2 and floxed-Grm5 mice. To demonstrate the role of synaptogenic molecules, we knockdowned them by delivering their siRNAs into S1.

Results: First, we validated astro-mGluR5-KO mice, and found that PSNL transiently upregulated mGluR5 in S1 astrocytes in Wt but not in astro-mGluR5-KO. Interestingly, mGluR5 was still increased in spinal astrocytes in astromGluR5-KO mice, indicating insufficient Cre-recombination in the spinal cord. Second, PSNL-induced mechanical allodynia was abolished in astro-mGluR5-KO mice, suggesting that upregulation of mGluR5 in S1 astrocytes but not in spinal astrocytes, should be required for allodynia. Third, mechanisms underlying astrocytic mGluR5-mediated allodynia were; (1) increase in Ca2+ fluctuations, (2) expression of synaptogenic molecules such as thrombospondin1, glypican4 and hevin, (3) synaptogenesis, (4) persistent rewiring of incorrect S1 circuits.

Conclusion: Re-appearance of mGluR5 in S1 astrocytes is essential for rewiring S1 cortical network, thereby leading to mechanical allodynia. We suggest that astrocytic mGluR5 in S1 can be a therapeutic target for neuropathic pain as well as a biomarker of synaptogenic astrocytes. 